OCHA-PP-106

NDA-FP-40

November 1997

\title{
Four-dimensional cosmology from dilaton coupled quantum matter in two dimensions
}

\author{
TомоKо KADOYOSHI

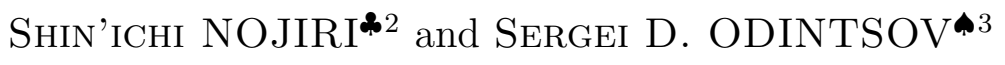 \\ Department of Physics, Ochanomizu University \\ Otsuka, Bunkyou-ku Tokyo 112, JAPAN \\ \& Department of Mathematics and Physics \\ National Defence Academy, Hashirimizu Yokosuka 239, JAPAN \\ \$ Tomsk Pedagogical University, 634041 Tomsk, RUSSIA \\ and \\ Dep.de Fisica, Universidad del Valle, AA25360, Cali, COLOMBIA
}

\begin{abstract}
The reduction of $4 \mathrm{D}$ Einstein gravity with $N$ minimal scalars leads to specific 2D dilaton gravity with dilaton coupled scalars. Applying $s$-wave and large $N$ approximation (where large $N$ quantum contribution due to dilaton itself is taken into account) we study 2D cosmology for CGHS model and for reduced Einstein gravity (in both cases with dilaton coupled scalars). Numerical study shows that in most cases we get 2D singular Universe which may correspond to big bang. Nevertheless, big crunch or non-singular Universes are also possible. For reduced $4 \mathrm{D}$ Einstein gravity one can regard the obtained 2D cosmology with time-dependent dilaton as 4D Kantowski-Sacks Universe of singular,non-singular or big crunch type.
\end{abstract}

PACS:

\footnotetext{
${ }^{1}$ e-mail: kado@fs.cc.ocha.ac.jp

2 e-mail : nojiri@cc.nda.ac.jp

3 e-mail : odintsov@quantum.univalle.edu.co,odintsov@kakuri2-pc.phys.sci.hiroshimau.ac.jp
} 
1. Introduction. It is quite well-known fact that models of two-dimensional dilaton gravity with matter may describe qualitatively the main properties of Hawking gravitational collapse [1] taking account of quantum matter back effects [2, 3] (for a review, see [4] and for a very incomplete list of earlier related works, see [5]). These models attract the interest being often exactly solvable ones and representing toy laboratory for study of Hawking radiation. From another point, the action of ref. [2]:

$$
S=\int d^{2} x \sqrt{-g}\left[-\frac{1}{16 \pi G} \mathrm{e}^{-2 \phi}\left(R+4(\nabla \phi)^{2}+4 \lambda^{2}\right)+\frac{1}{2} \sum_{i=1}^{N}\left(\nabla \chi_{i}\right)^{2}\right] .
$$

which belongs to more general class of renormalizable dilatonic gravities [6] may be relevant to description of radial modes of $4 \mathrm{D}$ extremal black holes [0].

However one can restrict $4 \mathrm{D}$ action of Einstein gravity with cosmological term and with matter described by $N$ minimal scalars $\chi_{i}$ to the metrics with spherical symmetry:

$$
d s^{2}=g_{\mu \nu} d x^{\mu} d x^{\nu}+\mathrm{e}^{-2 \phi} d \Omega
$$

Here two-dimensional metric and dilaton depend only from time and radius. Then spherically reduced action reads

$$
S_{\text {red }}=\int d^{2} x \sqrt{-g} \mathrm{e}^{-2 \phi}\left[-\frac{1}{16 \pi G}\left\{R+2(\nabla \phi)^{2}-2 \Lambda+2 \mathrm{e}^{2 \phi}\right\}+\frac{1}{2} \sum_{i=1}^{N}\left(\nabla \chi_{i}\right)^{2}\right] .
$$

Theory (31) represents the model which is completely different from (11) not only in gravitational sector but also in matter sector. Unlike to the previous models [1]-[5], one has to take into account quantum effects of dilaton coupled scalar and not of minimal scalars as in (11). Note that one could start from more general reduction having an arbitrary function $f(\phi)$ instead of $\mathrm{e}^{-2 \phi}$ in (22). In this case, one would have more general theory in two dimensions with the change $\mathrm{e}^{-2 \phi} \rightarrow f(\phi)$ in (3) and the correspondent change of dilaton dependent kinetic-like term in (3). Quantum field theory of dilaton coupled two-dimensional scalars (and spinors) with arbitrary dilaton function in curved spaces with dilaton has been first studied in ref. [8] where one-loop effective action has been found.

It is clear that it is action (3) which should be used for the study of reduced 4D theory from 2D dimensional point of view in $s$-wave and large $N$ 
approximation but not action (11). For that purpose, one needs the conformal anomaly for dilaton coupled scalars. Such anomaly for dilatonic coupling $\mathrm{e}^{-2 \phi}$ as well as correspondent anomaly induced effective action has been calculated by Bousso and Hawking [9]. For an arbitrary dilatonic coupling $f(\phi)$ the conformal anomaly and anomaly induced effective action has been found in ref. [10] (actually, this result is presented in non-explicit form already in ref. $[8])$.

Hawking radiation for 2D black holes with account of quantum effects of dilaton coupled scalars has been investigated in refs. [11, 12]. It is interesting to note in this respect that dilaton coupled spinor leads to the same trace anomaly as minimal spinor [8]. Using this fact, Hawking radiation in 2D dilatonic supergravity (for a review, see 13]) has been also discussed [14.

In the recent paper [15], quantum evolution of 4D black holes has been studied in $s$-wave and large $\mathrm{N}$ approximation taking account of dilaton coupled quantum matter. The possibility of anti-evaporation of black holes has been found.

In the present paper, we discuss another class of phenomena where quantum effects of dilaton coupled $2 D$ matter may be relevant, i.e., we discuss quantum cosmological models. We concentrate on the spherically symmetric metrics of Kantowski-Sachs form [16]:

$$
d s^{2}=g_{\mu \nu} d x^{\mu} d x^{\nu}+\mathrm{e}^{-2 \phi} d \Omega
$$

where $g_{\mu \nu}=a^{2}(t) \eta_{\mu \nu}$. Such metric (4) describes the Universe with a $S^{1} \times$ $S^{2}$ spatial geometry (for some its properties, see [17]). The back reaction problem for the time-dependent metric (4) in CGHS model with minimally coupled scalars has been studied in refs. [18]. Here, also 4D interpretation of results obtained has been presented.

However as it follows from above discussion, the correct approach to $4 \mathrm{D}$ quantum cosmology (4) in $s$-wave and large $N$ approximation is to start from the action (3) and take into account back-reaction via trace anomaly of dilaton coupled scalars. That is the purpose of present paper.

In order to see the difference from CGHS model with minimal scalars, we start in next section from CGHS model with dilaton coupled scalars. In this case, the model is not exactly solvable anymore. The numerical study of 2D time-dependent cosmology with time dependent dilaton is presented in large $N$ approximation. The comparison is done with results of refs. [18]. Note that such 2D cosmological quantum models may be of interest by itself. 
In the section 3 , we repeat the same study for $4 \mathrm{D}$ reduced theory with the action (3). We again discuss 2D cosmology. However, now 4D interpretation as Kantowski-Sacks models may be given.

2. CGHS model with dilaton coupled matter The starting point is Eq.(11). The large $N$ effective action produced by quantum dilaton coupled scalars (with account of quantum dilaton contribution) is 10

$$
\begin{aligned}
W= & -\frac{1}{2} \int d^{2} x \sqrt{-g}\left[\frac{N}{48 \pi} R \frac{1}{\Delta} R-\frac{1}{8 \pi}\left(\frac{f^{\prime 2}}{f}-f^{\prime \prime}\right)\left(\nabla^{\lambda} \chi_{i}\right)\left(\nabla_{\lambda} \chi_{i}\right) \frac{1}{\Delta} R\right. \\
& \left.-\frac{N}{16 \pi} \frac{f^{\prime 2}}{f^{2}} \nabla^{\lambda} \phi \nabla_{\lambda} \phi \frac{1}{\Delta} R-\frac{N}{8 \pi} \ln f R\right] .
\end{aligned}
$$

In the conformal gauge

$$
g_{ \pm \mp}=-\frac{1}{2} \mathrm{e}^{2 \rho}, \quad g_{ \pm \pm}=0
$$

the equations of motion which follow from Eq.(II) (where last ter is multiplied by $f(\phi)$ ) plus Eq.(5) are obtained by the variation over $g^{ \pm \pm}, g^{ \pm \mp}, \phi, \chi$

$$
\begin{aligned}
0= & \mathrm{e}^{-2 \phi}\left(4 \partial_{ \pm} \rho \partial_{ \pm} \phi-2\left(\partial_{ \pm} \phi\right)^{2}\right)+\frac{f}{2} \sum_{i}\left(\partial_{ \pm} \chi_{i}\right)^{2}+\frac{N}{12}\left(\partial_{ \pm}^{2} \rho-\partial_{ \pm} \rho \partial_{ \pm} \rho\right) \\
& +\frac{N}{8}\left\{\left(\partial_{ \pm} \tilde{\phi} \partial_{ \pm} \tilde{\phi}\right) \rho+\frac{1}{2} \frac{\partial_{ \pm}}{\partial_{\mp}}\left(\partial_{ \pm} \tilde{\phi} \partial_{\mp} \tilde{\phi}\right)\right\}+\frac{N}{8}\left\{-2 \partial_{ \pm} \rho \partial_{ \pm} \tilde{\phi}+\partial_{ \pm}^{2} \tilde{\phi}\right\} \\
& +\frac{1}{4} \sum_{i}\left\{g\left(\partial_{ \pm} \chi_{i}\right)^{2} \rho+\frac{1}{2} \frac{\partial_{ \pm}}{\partial_{\mp}}\left(g \partial_{ \pm} \chi_{i} \partial_{\mp} \chi_{i}\right)\right\}+t^{ \pm}\left(x^{ \pm}\right) \\
0 & \mathrm{e}^{-2 \phi}\left(2 \partial_{+} \partial_{-} \phi-4 \partial_{+} \phi \partial_{-} \phi-\lambda^{2} \mathrm{e}^{2 \rho}\right) \\
& -\frac{N}{12} \partial_{+} \partial_{-} \rho-\frac{N}{16} \partial_{+} \tilde{\phi} \partial_{-} \tilde{\phi}-\frac{N}{8} \partial_{+} \partial_{-} \tilde{\phi}-\frac{1}{8} g \sum_{i} \partial_{+} \chi_{i} \partial_{-} \chi_{i} \\
0 \quad & \mathrm{e}^{-2 \phi}\left(-4 \partial_{+} \partial_{-} \phi+4 \partial_{+} \phi \partial_{-} \phi+2 \partial_{+} \partial_{-} \rho+\lambda^{2} \mathrm{e}^{2 \rho}\right)+\frac{1}{4} f^{\prime} \sum_{i} \partial_{+} \chi_{i} \partial_{-} \chi_{i} \\
& -N \tilde{\phi}^{\prime}\left\{\frac{1}{16} \partial_{+}\left(\rho \partial_{-} \tilde{\phi}\right)+\frac{1}{16} \partial_{-}\left(\rho \partial_{+} \tilde{\phi}\right)-\frac{1}{8} \partial_{+} \partial_{-} \rho\right\} \\
& +\frac{1}{8} g^{\prime} \rho \sum_{i} \partial_{+} \chi_{i} \partial_{-} \chi_{i} \\
0 & \partial_{+}\left(f \partial_{-} \chi_{i}\right)+\partial_{-}\left(f \partial_{+} \chi_{i}\right)-\frac{1}{2}\left\{\partial_{+}\left(g \rho \partial_{-} \chi_{i}\right)+\partial_{-}\left(g \rho \partial_{+} \chi_{i}\right)\right\}
\end{aligned}
$$


Here $\tilde{\phi}=\ln f, g=\frac{f^{\prime 2}}{f}-f^{\prime \prime}$ and $t^{ \pm}\left(x^{ \pm}\right)$is a function which is determined by the boundary condition. When we consider the cosmological problem, we can assume that all the fields depend only on time $t$ and replace $\partial_{ \pm} \rightarrow \frac{1}{2} \partial_{t}$. Then Eq.(7) tells $t^{ \pm}$is a constant: $t^{ \pm}=\frac{N}{4} t_{0}$ and we obtain

$$
\begin{aligned}
0= & \mathrm{e}^{-2 \phi}\left(4 \partial_{t} \rho \partial_{t} \phi-2\left(\partial_{t} \phi\right)^{2}\right)+\frac{f}{2} \sum_{i}\left(\partial_{t} \chi_{i}\right)^{2} \\
& +\frac{N}{12}\left(\partial_{t}^{2} \rho-\partial_{t} \rho \partial_{t} \rho\right)+\frac{N}{8}\left(\rho+\frac{1}{2}\right) \partial_{t} \tilde{\phi} \partial_{t} \tilde{\phi} \\
& +\frac{N}{8}\left\{-2 \partial_{t} \rho \partial_{t} \tilde{\phi}+\partial_{t}^{2} \tilde{\phi}\right\}+\frac{1}{4}\left(\rho+\frac{1}{2}\right) \sum_{i} g\left(\partial_{t} \chi_{i}\right)^{2}+N t_{0} \\
0= & \mathrm{e}^{-2 \phi}\left(2 \partial_{t} \partial_{t} \phi-4 \partial_{t} \phi \partial_{t} \phi-4 \lambda^{2} \mathrm{e}^{2 \rho}\right) \\
& -\frac{N}{12} \partial_{t}^{2} \rho-\frac{N}{16} \partial_{t} \tilde{\phi} \partial_{t} \tilde{\phi}-\frac{N}{8} \partial_{t}^{2} \tilde{\phi}-\frac{1}{8} g \sum_{i} \partial_{t} \chi_{i} \partial_{t} \chi_{i} \\
0 & \mathrm{e}^{-2 \phi}\left(-4 \partial_{t} \partial_{t} \phi+4 \partial_{t} \phi \partial_{t} \phi+2 \partial_{t} \partial_{t} \rho+4 \lambda^{2} \mathrm{e}^{2 \rho}\right)+\frac{1}{4} f^{\prime} \sum_{i} \partial_{t} \chi_{i} \partial_{t} \chi_{i} \\
& -\frac{N}{8} \varphi^{\prime}\left\{\partial_{t}\left(\rho \partial_{t} \tilde{\phi}\right)-\partial_{t}^{2} \rho\right\}+\frac{1}{8} g^{\prime} \rho \sum_{i} \partial_{t} \chi_{i} \partial_{t} \chi_{i} \\
0= & \partial_{t}\left\{\left(f-\frac{1}{2} g \rho\right) \partial_{t} \chi_{i}\right\} .
\end{aligned}
$$

Eq.(14) can be integrated to be $\left(f-\frac{1}{2} g \rho\right) \partial_{t} \chi_{i}=a_{i}$. Here $a_{i}$ is a constant of the integration.

When we investigate the cosmological problem, it is often more convenient to use the gauge where $g_{t t}=-1$ and $g_{t x}=g_{x t}=0$ than to use the conformal gauge (6). Since the metric under consideration depend only on $t$, the change of the gauge choice from conformal gauge is given by $d \hat{t}=\mathrm{e}^{\rho} d t$, accordingly

$$
\partial_{t}=\mathrm{e}^{\rho} \partial_{\hat{t}}, \quad \partial_{t}^{2}=\mathrm{e}^{2 \rho}\left(\partial_{\hat{t}}^{2}+\left(\partial_{\hat{t}} \rho\right) \partial_{\hat{t}}\right) .
$$

In the following we call $t$ as the conformal time and $\hat{t}$ as the cosmological time.

For $f(\phi)=\mathrm{e}^{-2 \phi}(\tilde{\phi}=-2 \phi)$, we find $g=0$ and the equations are reduced to be

$$
0=\mathrm{e}^{-2 \phi}\left(4 \partial_{t} \rho \partial_{t} \phi-2\left(\partial_{t} \phi\right)^{2}+\frac{1}{2} \sum_{i}\left(\partial_{t} \chi_{i}\right)^{2}\right)+\frac{N}{12}\left(\partial_{t}^{2} \rho-\left(\partial_{t} \rho\right)^{2}\right)
$$




$$
\begin{aligned}
& +\frac{N}{2}\left(\rho+\frac{1}{2}\right)\left(\partial_{t} \phi\right)^{2}+\frac{N}{4}\left\{2 \partial_{t} \rho \partial_{t} \phi-\partial_{t}^{2} \phi\right\}+N t_{0} \\
0= & \mathrm{e}^{-2 \phi}\left(2 \partial_{t}^{2} \phi-4\left(\partial_{t} \phi\right)^{2}-4 \lambda^{2} \mathrm{e}^{2 \rho}\right)-\frac{N}{12} \partial_{t}^{2} \rho-\frac{N}{4}\left(\partial_{t} \phi\right)^{2}+\frac{N}{4} \partial_{t}^{2} \phi \\
0= & \mathrm{e}^{-2 \phi}\left(-4 \partial_{t}^{2} \phi+4\left(\partial_{t} \phi\right)^{2}+2 \partial_{t}^{2} \rho+4 \lambda^{2} \mathrm{e}^{2 \rho}-\frac{1}{2} \sum_{i}\left(\partial_{t} \chi_{i}\right)^{2}\right) \\
& +\frac{N}{4}\left\{-2 \partial_{t}\left(\rho \partial_{t} \phi\right)-\partial_{t}^{2} \rho\right\} \\
0= & \partial_{t}\left\{\mathrm{e}^{-2 \phi} \partial_{t} \chi_{i}\right\}
\end{aligned}
$$

and

$$
\partial_{t} \chi_{i}=a_{i} \mathrm{e}^{2 \phi}
$$

First we consider $N \rightarrow \infty$ case where the equations become simpler:

$$
\begin{aligned}
0= & \frac{a^{2}}{2} \mathrm{e}^{2 \phi}+\frac{1}{12}\left(\partial_{t}^{2} \rho-\left(\partial_{t} \rho\right)^{2}\right)+\frac{1}{2}\left(\rho+\frac{1}{2}\right)\left(\partial_{t} \phi\right)^{2} \\
& +\frac{1}{4}\left\{2 \partial_{t} \rho \partial_{t} \phi-\partial_{t}^{2} \phi\right\}+t_{0} \\
0= & -\frac{1}{12} \partial_{t}^{2} \rho-\frac{1}{4}\left(\partial_{t} \phi\right)^{2}+\frac{1}{4} \partial_{t}^{2} \phi \\
0= & \frac{a^{2}}{2} \mathrm{e}^{2 \phi}+\frac{1}{4}\left\{-2 \partial_{t}\left(\rho \partial_{t} \phi\right)-\partial_{t}^{2} \rho\right\} .
\end{aligned}
$$

Here we used Eq.(20) and $a^{2} \equiv \frac{1}{N} \sum_{i} a_{i}^{2}$. Combining (21) and (22), we obtain

$$
0=-\frac{1}{12}\left(\partial_{t} \rho\right)^{2}+\frac{1}{2} \rho\left(\partial_{t} \phi\right)^{2}+\frac{1}{2} \partial_{t} \rho \partial_{t} \phi+t_{0}+\frac{a^{2}}{2} \mathrm{e}^{2 \phi} \text {. }
$$

When $t_{0}=a=0$, we find

$$
\partial_{t} \phi=\frac{1}{2 \rho}\left\{-1 \pm \sqrt{1+\frac{2}{3} \rho}\right\} \partial_{t} \rho
$$

that is,

$$
\phi=\int \frac{d \rho}{2 \rho}\left\{-1 \pm \sqrt{1+\frac{2}{3} \rho}\right\}
$$


Substituting Eq.(25) into Eq.(23), we obtain

$$
\rho=-\frac{3}{2}+(\alpha t+\beta)^{\frac{2}{3}} .
$$

Here $\alpha$ and $\beta$ are the constant of the integration. The solution (27) has the curvature singularity when $\alpha t+\beta=0$. Since $\rho$ is monotonically increasing function with respect to $t$ (and also the cosmological time $\hat{t}$ since $\mathrm{e}^{\rho}$ is positive) when $\alpha>0$, the solution (27) can express the expanding universe solution where the big bang is given by the curvature singularity at $\alpha t+\beta=0$.

We now consider Eqs.(21), (22) and (23) numerically. If we define new variables $P$ and $R$ by

$$
P=\partial_{t} \phi, \quad R=\partial_{t} \rho
$$

Eqs. (21) and (22) give

$$
\begin{aligned}
& \partial_{t} P=\left(1+\frac{2}{3} \rho\right)^{-1}\left(P^{2}-\frac{2}{3} R P-\frac{2}{3} a^{2} \mathrm{e}^{2 \phi}\right) \\
& \partial_{t} R=-2\left(1+\frac{2}{3} \rho\right)^{-1}\left(\rho P^{2}+R P+a^{2} \mathrm{e}^{2 \phi}\right)
\end{aligned}
$$

and Eq.(24) can be rewritten as

$$
0=Q \equiv-\frac{1}{6} R^{2}+\rho P^{2}+R P+2 t_{0}+a^{2} \mathrm{e}^{2 \phi}
$$

Using Eq.(31) we can rewrite Eq.(30) as follows:

$$
\partial_{t} R=-\left(1+\frac{2}{3} \rho\right)^{-1}\left(\frac{1}{3} R^{2}-4 t_{0}\right) .
$$

Eqs.(29) and (30) tell that there is a singularity when $\rho=\rho_{c} \equiv-\frac{3}{2}$. A special case of the singularity is that of (27). Therefore this singularity can be interpreted to be the singularity of the big-bang.

Eq.(31) can be regarded as the constraint for the initial values of $\phi, \rho, P$ and $R$. We can directly check that $\partial_{t} Q=0$ by using (29) and (30). Then we solve the equations (28), (29) and (30) choosing the initial values of $\phi, \rho$, $P$ and $R$ satisfying Eq.(31). Note that also Eq.(32) does not contain $\phi$ but only $\rho$. 
Therefore if we are interested in the behavior of $\rho$, that is, the timedevelopment of the universe, we only need to solve Eq.(32). If we define a new variable $\hat{\rho}$ by $\hat{\rho}=\left(\rho+\frac{3}{2}\right)^{\frac{3}{2}}$, we can rewrite (32) as follows

$$
\partial_{t}^{2} \hat{\rho}=9 t_{0} \hat{\rho}^{-\frac{1}{3}} .
$$

Eq.(33) can be compared with the Newton's equation of motion of a particle with unit mass in the potential $V(\hat{\rho})$ given by

$$
V(\hat{\rho})=-\frac{27}{4} t_{0} \hat{\rho}^{\frac{4}{3}} .
$$

Therefore the expanding universe turns to shrink if $t_{0}<0$ as in the Friedmann universe with $\Omega>0$. On the other hand, if $t_{0}>0$, the universe continues to expand as in $\Omega<0$.

Numerical study of Eq.(32) is given in Figures. The vertical line corresponds to $\rho$ and the horizontal line to $t$. Fig. 1 represents the case $\rho(0)=-1.4$, $\dot{\rho}(0)=1$, and $t_{0}=1$ (solid line), $t_{0}=0$ (dashed line) and $t_{0}=-1$ (dotdashed line). In Fig.2, the horizontal line is given by the cosmolgical time $\hat{t}$ in (15). Fig.2 also represents the case $\rho(0)=-1.4, \dot{\rho}(0)=1$, and $t_{0}=1$ (solid line), $t_{0}=0$ (dashed line) and $t_{0}=-1$ (dot-dashed line).

We now consider the finite $N$ case. Using (16) and (17), we obtain

$$
\begin{aligned}
\partial_{t}^{2} \phi= & -2\left(1+\frac{N}{8} \mathrm{e}^{2 \phi}\right) \partial_{t} \rho \partial_{t} \phi+3\left(1-\frac{N}{12} \mathrm{e}^{2 \phi} \rho\right)\left(\partial_{t} \phi\right)^{2} \\
& +\frac{N}{24} \mathrm{e}^{2 \phi}\left(\partial_{t} \rho\right)^{2}-\frac{N}{4} a^{2} \mathrm{e}^{4 \phi}+2 \lambda^{2} \mathrm{e}^{2 \rho}-\frac{1}{2} \mathrm{e}^{2 \phi} N t_{0} \\
\partial_{t}^{2} \rho= & 6\left\{\left(1+\frac{4}{N} \mathrm{e}^{-2 \phi}\right)-\left(1+\frac{N}{8} \mathrm{e}^{2 \phi}\right) \rho\right\}\left(\partial_{t} \phi\right)^{2} \\
& -\frac{48}{N} \mathrm{e}^{-2 \phi}\left(1+\frac{N}{8} \mathrm{e}^{2 \phi}\right)^{2} \partial_{t} \rho \partial_{t} \phi+\left(1+\frac{N}{8} \mathrm{e}^{2 \phi}\right)\left(\partial_{t} \rho\right)^{2} \\
& +6 \lambda^{2} \mathrm{e}^{2 \rho}-6\left(1+\frac{N}{8} \mathrm{e}^{2 \phi}\right) a^{2} \mathrm{e}^{2 \phi}-12\left(1+\frac{N}{8} \mathrm{e}^{2 \phi}\right) t_{0} .
\end{aligned}
$$

If we delete the terms in (16), (17) and (18) which contain the second order derivatives, we obtain

$0=\left\{-\frac{96}{N} \mathrm{e}^{-4 \phi}-4 \mathrm{e}^{-2 \phi}+2 N+\frac{3 N^{2}}{16} \mathrm{e}^{2 \phi}+N\left(1+\frac{N}{8} \mathrm{e}^{2 \phi}\right) \rho\right\} \partial_{t} \rho \partial_{t} \phi$ 


$$
\begin{aligned}
& +\left\{-\frac{3 N}{2}-2 \mathrm{e}^{-2 \phi}+\frac{48}{N} \mathrm{e}^{-4 \phi}-\frac{1}{2}\left(N+24 \mathrm{e}^{-2 \phi}-\frac{3 N^{2}}{8} \mathrm{e}^{2 \phi}\right) \rho\right. \\
& \left.+\frac{N^{2}}{8} \mathrm{e}^{2 \phi} \rho^{2}\right\}\left(\partial_{t} \phi\right)^{2}+\left(-\frac{N}{6}+2 \mathrm{e}^{-2 \phi}-\frac{N^{2}}{32} \mathrm{e}^{2 \phi}-\frac{N^{2}}{48} \mathrm{e}^{2 \phi} \rho\right)\left(\partial_{t} \rho\right)^{2} \\
& +\left(8 \mathrm{e}^{-2 \phi}-N \rho-\frac{3 N}{2}\right) \lambda^{2} \mathrm{e}^{2 \rho}+2 N\left(1+\frac{3 N}{16} \mathrm{e}^{2 \phi}-\frac{12}{N} \mathrm{e}^{-2 \phi}+\frac{N}{8} \rho \mathrm{e}^{2 \phi}\right) t_{0} \\
& +\left(\frac{3 N}{4}+2 \mathrm{e}^{-2 \phi}-\frac{48}{N} \mathrm{e}^{-4 \phi}+\frac{N}{2} \rho\right) \frac{N a^{2}}{4} \mathrm{e}^{4 \phi} .
\end{aligned}
$$

Choosing the initial condition which satisfies (37), we can solve (35) and (36). Otherwise (37) can be regarded to be the equation which determines $t_{0}$ from the initial value. Note that when $a=\lambda^{2}=0$, (35) and (36) have trivial solutions where $\rho$ and $\phi$ are constants $\left(t_{0}=0\right)$. $t=0$

We have calculated numericaly several cases with the initial condition at

$$
\phi=\dot{\phi}=\rho=\dot{\rho}=0 .
$$

When $\lambda^{2}<0$, $\phi$ and $\rho$ decrease monotonically in most of cases. A typical example is given in Fig.3. $\phi$ is given in solid line and $\rho$ in dashed line. The parameters in Fig.3 are chosen to be $N=1, a=1, \lambda^{2}=-1$ and $t_{0}=-0.823699$. When $\lambda^{2}>0, \phi$ increases monotonically and $\rho$ increases first and decreases in most of cases. A typical example is also given in Fig.4 ( $\phi$ is solid line and $\rho$ dashed line) where the parameter are chosen to be $N=1, a=1, \lambda^{2}=1$ and $t_{0}=-0.141791$. In the above two cases of Fig.3 and Fig.4, there appear singularities in the finite conformal time $t$. The singularities occur when $\rho$ is decreasing, what tells that the singularities occur in the finite cosmological time $\hat{t}$ in Eq.(15). Since the system is, of course, invariant if we change $t$ with $-t$, the singularity can be regarded to express the big bang if we reverse the direction of the time $t$. Due to the initial condition (38), we can paste the solutions in Figs.3,4 with the time reversed solution at $t=0$. The combined solution would express the process that the universe generated by the big bang disappears by the big crunch. The obtained solution would correspond to $n>1$ case in [18.

We also find a solution without singularity, which is given in Fig.5 ( $\phi$ is solid line and $\rho$ dashed line) where the parameter are chosen to be $N=1$, $a=1, \lambda^{2}=0$ and $t_{0}=-0.523121$. In Fig.5, $\phi$ oscillates like sine or cosine function and $\rho$ decreases slowly with vibration. Such a vibrating 
solutions also appear when $N$ is large. In Fig.6 ( $\phi$ is solid line and $\rho$ dashed line) where the parameters are chosen to be $N=100, a=1, \lambda^{2}=1$ and $t_{0}=-0.487264$. The solution in Fig. 6 has a singularity at the finite conformal and/or cosmological time. Any solution which shows the behavior like in Fig.5 and Fig.6 does not seen in 18 .

In the above solutions, if we exchange the role of time and radius, we obtain a static object, which can be regarded as a kind of black hole.

3. The reduced model. Two-dimensional dilaton gravity with scalars can be regarded as the dimensional reduction of four dimensional Einstein gravity whose metric is given by

$$
d s^{2}=\mathrm{e}^{2 \rho}\left(-d t^{2}+d r^{2}\right)+\mathrm{e}^{-2 \phi} d \Omega^{2} .
$$

The scalar curvature corresponding to the metric (39) is given by

$$
R_{4 d}=-\mathrm{e}^{-2 \rho}\left\{2 \partial_{t}^{2} \rho-4 \partial_{t}^{2} \phi+6\left(\partial_{t} \phi\right)^{2}\right\} .
$$

The obtained reduced Einstein-scalar action has the form of Eq.(3). Adding to it the quantum correction obtained from conformal anomaly we may get the following equations of motion similar to (35) and (36):

$$
\begin{aligned}
\partial_{t}^{2} \phi= & -2\left(1+\frac{N}{8} \mathrm{e}^{2 \phi}\right) \partial_{t} \rho \partial_{t} \phi+\left(\frac{5}{2}-\frac{N}{4} \mathrm{e}^{2 \phi} \rho\right)\left(\partial_{t} \phi\right)^{2} \\
& +\frac{N}{24} \mathrm{e}^{2 \phi}\left(\partial_{t} \rho\right)^{2}-\frac{N}{4} a^{2} \mathrm{e}^{4 \phi}+\lambda^{2} \mathrm{e}^{2 \rho}+\mathrm{e}^{2 \phi+2 \rho}-\frac{1}{2} \mathrm{e}^{2 \phi} N t_{0} \\
\partial_{t}^{2} \rho= & 6\left\{\left(\frac{1}{4}+\frac{3}{N} \mathrm{e}^{-2 \phi}\right)-\left(1+\frac{N}{8} \mathrm{e}^{2 \phi}\right) \rho\right\}\left(\partial_{t} \phi\right)^{2} \\
& -\frac{48}{N} \mathrm{e}^{-2 \phi}\left(1+\frac{N}{8} \mathrm{e}^{2 \phi}\right)^{2} \partial_{t} \rho \partial_{t} \phi+\left(1+\frac{N}{8} \mathrm{e}^{2 \phi}\right)\left(\partial_{t} \rho\right)^{2} \\
& +3 \lambda^{2} \mathrm{e}^{2 \rho}+3 \mathrm{e}^{2 \phi+2 \rho}-6\left(1+\frac{N}{8} \mathrm{e}^{2 \phi}\right) a^{2} \mathrm{e}^{2 \phi}-12\left(1+\frac{N}{8} \mathrm{e}^{2 \phi}\right) t_{0} .
\end{aligned}
$$

We also obtain the equation for the initial condition corresponding to (37):

$$
\begin{aligned}
0= & \left\{-\frac{96}{N} \mathrm{e}^{-4 \phi}-8 \mathrm{e}^{-2 \phi}+2 N+\frac{3 N^{2}}{16} \mathrm{e}^{2 \phi}+N\left(1+\frac{N}{8} \mathrm{e}^{2 \phi}\right) \rho\right\} \partial_{t} \rho \partial_{t} \phi \\
& +\left\{-\frac{9 N}{8}+3 \mathrm{e}^{-2 \phi}+\frac{24}{N} \mathrm{e}^{-4 \phi}-\frac{1}{2}\left(\frac{3}{2} N+24 \mathrm{e}^{-2 \phi}-\frac{3 N^{2}}{8} \mathrm{e}^{2 \phi}\right) \rho\right.
\end{aligned}
$$




$$
\begin{aligned}
& \left.+\frac{N^{2}}{8} \mathrm{e}^{2 \phi} \rho^{2}\right\}\left(\partial_{t} \phi\right)^{2}+\left(-\frac{N}{12}+2 \mathrm{e}^{-2 \phi}-\frac{N^{2}}{32} \mathrm{e}^{2 \phi}-\frac{N^{2}}{48} \mathrm{e}^{2 \phi} \rho\right)\left(\partial_{t} \rho\right)^{2} \\
& -\left(-6 \mathrm{e}^{-2 \phi}+\frac{N}{2} \rho+\frac{3 N}{4}\right) \lambda^{2} \mathrm{e}^{2 \rho}-\left(-4 \mathrm{e}^{-2 \phi}+\frac{N}{2} \rho+\frac{3 N}{4}\right) \mathrm{e}^{2 \phi+2 \rho} \\
& +2 N\left(\frac{1}{2}+\frac{3 N}{8} \mathrm{e}^{2 \phi}-\frac{12}{N} \mathrm{e}^{-2 \phi}+\frac{N}{16} \rho \mathrm{e}^{2 \phi}\right) t_{0} \\
& +\left(\frac{3 N}{4}-\frac{48}{N} \mathrm{e}^{-4 \phi}+\frac{N}{2} \rho\right) \frac{N a^{2}}{4} \mathrm{e}^{4 \phi} .
\end{aligned}
$$

Here $\lambda^{2}=-\Lambda$. The behaviors of the numerical solutions of the equations (41), (42) and (43) with the initial condition (38) are very similar to those of CGHS type model. When $\lambda^{2}<0, \phi$ and $\rho$ decrease monotonically in most of cases as in Fig.3. A typical example is given in Fig.7 where the parameters are chosen to be $N=1, a=0$ and $\lambda^{2}=-1$. in Fig.7, solid line and dashed line represent $\phi$ and $\rho$ respectively and dot-dashed line shows the behaviour of the $4 \mathrm{~d}$ scalar curvature $R_{4 d}$ in (40). When $\lambda^{2}>0, \phi$ increases monotonically and $\rho$ increases first and decreases in most of cases. A typical example is also given in Fig.8 (solid line, dashed line and dot-dashed line represent $\phi$, $\rho$ and $R_{4 d}$, respectively) where the parameters are chosen to be $N=1$, $a=0, \lambda^{2}=1$. In the above two cases of Fig. 7 and Fig.8, there also appear singularities in the finite conformal time $t$ and/or in the finite cosmological time $\hat{t}$. Vibrating solutions also appear when $N$ is large. In Fig.9 (solid line, dashed line and dot-dashed line represent $\phi, \rho$ and $R_{4 d}$, respectively) where the parameters are chosen to be $N=100, a=1$ and $\lambda^{2}=1$. The solution in Fig.9 has a singularity at the finite conformal and/or cosmological time. Since $\rho$ becomes small at the singularity, the singularity would correspond to the big crunch. As in case of CGHS model with dilaton coupled scalars in the previous section, we can paste the solutions in Figs.7, 8, 9 with the time reversed solution at $t=0$. The combined solution would also express the process that the universe generated by the big bang disappears by the big crunch. Especially the cases of Fig.7 and Fig.8 would correspond to $n=1$ case in 18 but the solution corresponding to Fig.9 shows rather different behavior.

We can also regard the solution as the Kantowski-Sacks Universe which has a $S^{1} \times S^{2}$ spatial geometry in (4) and the metric (39). Then the solution of Fig. 7 expresses the universe where $S^{1}$ crunches to become a point but the radius of $S^{2}$ diverges in the finite time. In the solution of Fig.7, the $4 \mathrm{~d}$ scalar 
curvature diverges positively. On the other hand, the solutions of Fig.8 and 9 express the universe where both of $S^{1}$ and $S^{2}$ crunch. In the solution of Fig.8, the $4 \mathrm{~d}$ scalar curvature diverges negatively at the singularity. Remarkably, the $4 \mathrm{~d}$ scalar curvature vanishes and is not singular at the singularity in the solution of Fig.9. That indicates to a possibility of constructing new versions of topologically non-trivial inflationary Universe.

\section{References}

[1] S.W. Hawking, Comm.Math.Phys. 43 (1975) 199.

[2] C.G. Callan, S.B. Giddings, J.A. Harvey and A. Strominger, Phys. Rev. D45 (1992) 1005.

[3] J.G. Russo, L. Susskind and L. Thorlacius, Phys.Lett. B292 (1992) 13; Phys.Rev. D47 (1993) 533.

[4] T. Banks, Spring School on Supersymmetry and Superstrings, hepth/9412139; A. Strominger, Les Houches lectures on black holes, hepth/9501071; S. Giddings, Summer School in High Energy Physics and Cosmology, Trieste, hep-th/9412138; M.O. Katanaev, W. Kummer, H. Liebl and D.V. Vassilevich, preprint TUW-97-10, 1997.

[5] S.P. de Alwis, Phys.Lett. B289 (1992) 278; A. Bilal and C. Callan, Nucl.Phys. B394 (1993) 73; S. Nojiri and I. Oda, Phys.Lett. B294 (1992) 317, Nucl.Phys. B406 (1993) 499; T. Banks, A. Dabholkar, M. Douglas and M. O'Loughlin, Phys.Rev. D45 (1992) 3607; R.B. Mann, Phys.Rev. D47 (1993) 4438; E. Elizalde and S.D. Odintsov, Nucl.Phys. B399 (1993) 581; D. Louis-Martinez and G. Kunstatter, Phys.Rev. D49 (1994) 5227. G. Amelino-Camelia, L. Griguolo and D. Seminara, Phys.Lett. B371 (1996) 41; S. Bose, L. Parker and Y. Peleg, Phys.Rev. D52 (1995) 3512; T. Stroble, Phys.Rev. D50 (1994) 3961; D. Cangemi and R. Jackiw, Phys.Rev. D50 (1994) 3913; D.Louis-Martinez, J. Gegenberg and G. Kunstatter, Phys.Lett. B321 (1994) 193.

[6] S.D. Odintsov and I.L. Shapiro, Phys. Lett. B263 (1991) 183; Mod. Phys. Lett. A7 (1992) 437; Int. J. Mod. Phys. D1 (1993)571. 
[7] G.W. Gibbons, Nucl.Phys. B207 (1982) 337; G.W. Gibbons and K. Maeda, Nucl.Phys. B298 (1988) 741; S.B. Giddings and A. Strominger, Phys.Rev.Lett. 67 (1991) 1930; D. Garfinkle, G.T. Horowitz and A. Strominger, Phys.Rev. D43 (1991) 3140.

[8] E. Elizalde, S. Naftulin and S.D. Odintsov, Phys. Rev. D49 (1994) 2852.

[9] R. Bousso and S.W. Hawking, preprint hep-th/9705236.

[10] S. Nojiri and S.D. Odintsov, hep-th/9706009; Mod.Phys.Lett. A12 (1997) 2083.

[11] S. Nojiri and S.D. Odintsov, hep-th/9706143.

[12] W. Kummer, H. Liebl and D. Vassilevich, hep-th/9707041.

[13] S.J. Gates, M.T. Grisaru, M. Rocek and W. Siegel, "Superspace or One Thousand and One Lessons in Supersymmetry", Benjamin/cummings (1983) (Frontiers in Physics, 58).

[14] S. Nojiri and S.D. Odintsov, hep-th/9708139, Phys.Lett.B to appear.

[15] R. Bousso and S. Hawking, hep-th/9709224.

[16] R. Kantowski and R. Sacks, J.Math.Phys. 7 (1967) 2315;

[17] R. Laflamme and E.P. Shellard, Phys.Rev. D35 (1987); J. Louko and T. Vachaspati, Phys.Lett. B223 (1989) 21.

[18] A. Fabbri and J. Russo, Phys.Rev. D53 (1996) 6995, J. Russo and F. Mazzitelli, Phys. Rev. D47 (1993) 4490. 


\section{Figure Captions}

Fig.1 Conformal time $t$ (horizontal line) versus $\rho$ (vertical line) for $t_{0}=1$ (solid line), $t_{0}=0$ (dashed line) and $t_{0}=-1$ (dot-dashed line).

Fig.2 Cosmological time $\hat{t}$ (horizontal line) versus $\rho$ (vertical line) for $t_{0}=1$ (solid line), $t_{0}=0$ (dashed line) and $t_{0}=-1$ (dot-dashed line).

Fig. $3 t$ versus $\phi$ (solid line) and $\rho$ (dashed line) for $N=1, a=1, \lambda^{2}=-1$ and $t_{0}=-0.823699$.

Fig. $4 t$ versus $\phi$ (solid line) and $\rho$ (dashed line) for $N=1, a=1, \lambda^{2}=1$ and $t_{0}=-0.141791$.

Fig.5 $t$ versus $\phi$ (solid line) and $\rho$ (dashed line) for $N=1, a=1, \lambda^{2}=0$ and $t_{0}=-0.523121$.

Fig. $6 t$ versus $\phi$ (solid line) and $\rho$ (dashed line) for $N=100, a=1, \lambda^{2}=1$ and $t_{0}=-0.487264$.

Fig.7 $t$ versus $\phi$ (solid line), $\rho$ (dashed line) and $R_{4 d}$ for $N=1, a=0$, $\lambda^{2}=1$.

Fig. $8 t$ versus $\phi$ (solid line), $\rho$ (dashed line) and $R_{4 d}$ for $N=1, a=0$, $\lambda^{2}=1$.

Fig.9 $t$ versus $\phi$ (solid line), $\rho$ (dashed line) and $R_{4 d}$ for $N=100, a=1$ and $\lambda^{2}=1$. 


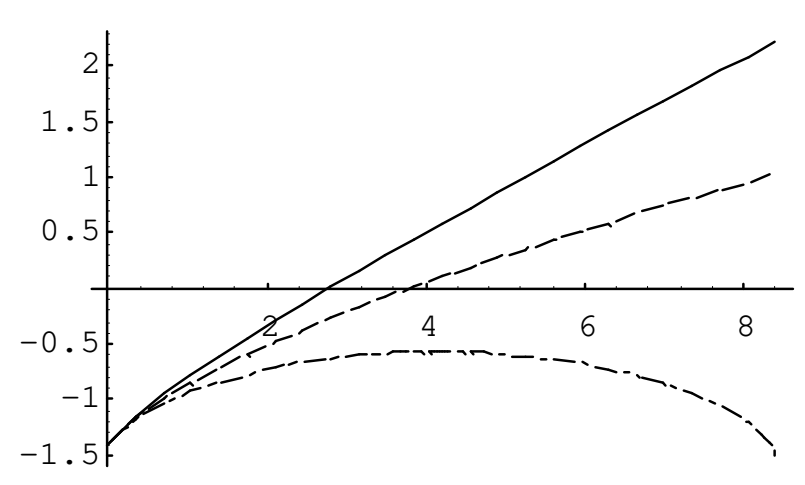

Fig.1

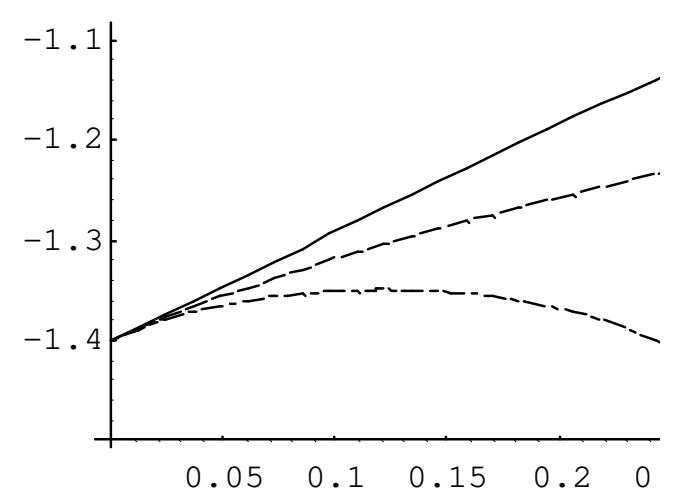

Fig.2

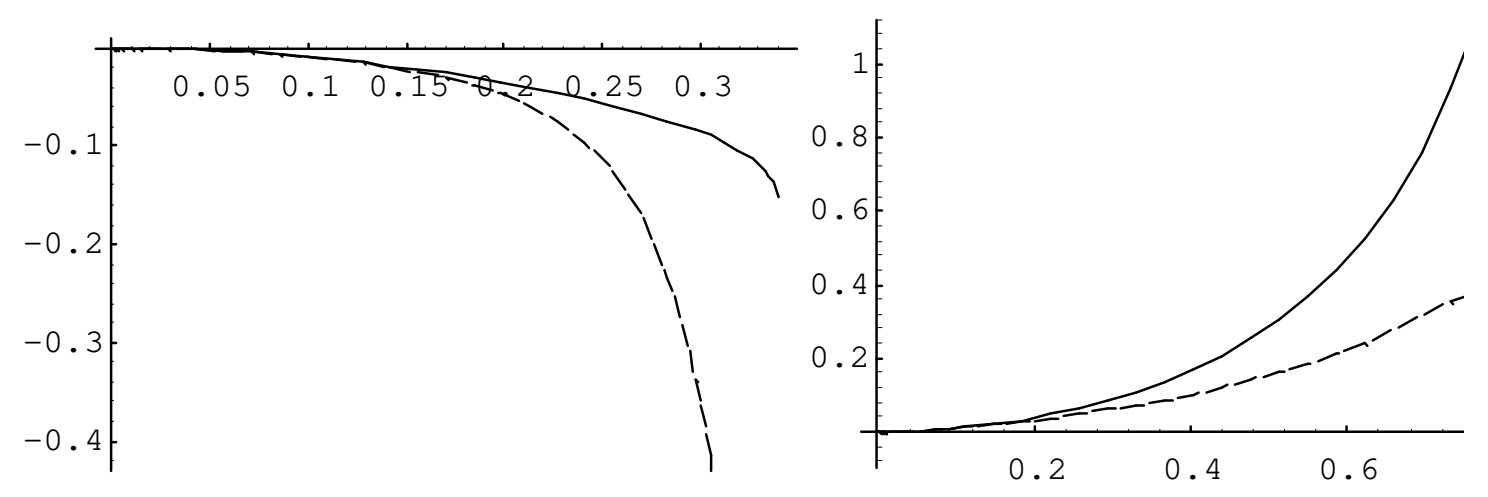

Fig.3

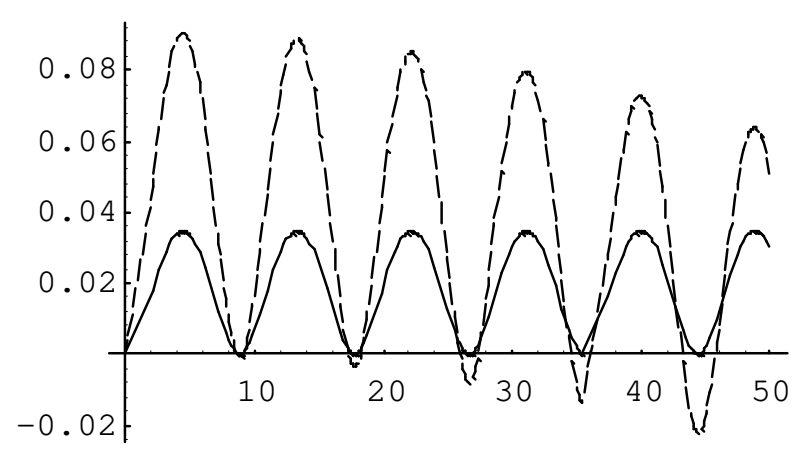

Fig.5

Fig.4

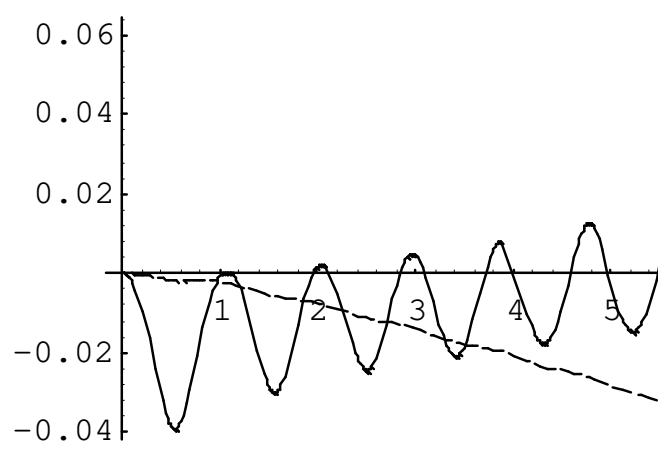

Fig.6 


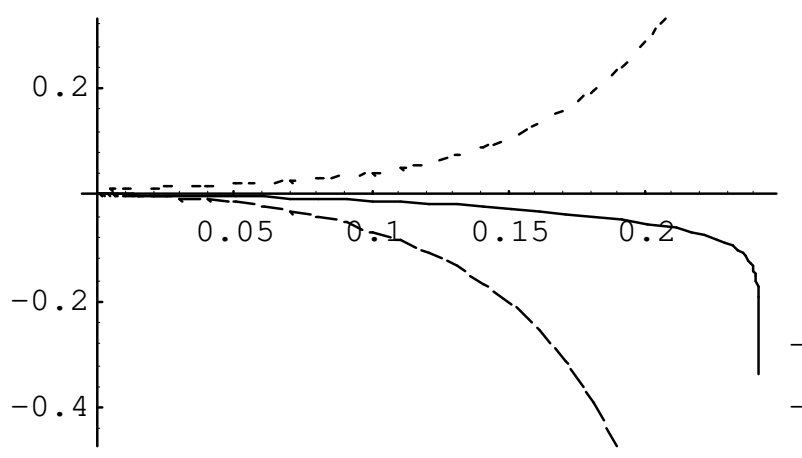

Fig.7



Fig.9



Fig.8 